\title{
Role of Computed Tomography Scan in Evaluating Children with Seizures
}

\author{
${ }^{1}$ Vasudha Sipayya, ${ }^{2}$ Shivali V Kashikar
}

\begin{abstract}
Introduction: Seizures are a common cause of morbidity and mortality in childhood. It is important to establish the cause of seizures to appropriately manage such patients. The etiology of seizures is different in developing countries as compared to the developed world. Computed tomography (CT) scan is easily available, is less sensitive to patient motion, and does not generally require sedation. Also the cost is less as compared to MRI, and this makes it an important screening tool in developing countries like India.
\end{abstract}

Aims: To determine the diagnostic ability of CT to determine the cause of seizures in children.

\section{Settings and design: A prospective analytical study.}

Materials and methods: Computed tomography scans of 84 children presenting with seizures during the period of September 2014 to August 2015 were analyzed. The study was done using 16-slice CT scan. Intravenous contrast was administered as needed. Uncooperative patients were sedated for the scan.

Results: Out of 84 scans, 40 scans (47.65\%) were found to be abnormal. Maximum number of patients were found to have congenital anomalies (10), followed by hydrocephalus which was found in nine children. Five children had posttraumatic seizures. Four presented with tumors. Four children showed features of hypoxic ischemic encephalopathy. Three each had granuloma and calcification. Two children with seizures had infectious causes, which were clinically diagnosed to be meningitis.

Conclusion: Our study indicates that CT is an extremely useful tool for screening the patients of seizures. It is also useful in identifying the treatable causes and monitoring the progression. It is recommended that all children with seizures should be evaluated with CT scan.

Keywords: Children, Computed tomography scan, Congenital anomalies, Hydrocephalus, Seizures.

How to cite this article: Sipayya V, Kashikar SV. Role of Computed Tomography Scan in Evaluating Children with Seizures. Int J Recent Surg Med Sci 2016;2(1):15-18.

Source of support: Nil

Conflict of interest: None

\footnotetext{
${ }^{1}$ Resident, ${ }^{2}$ Professor

1,2Department of Radiodiagnosis, Datta Meghe Institute of Medical Sciences, Wardha, Maharashtra, India

Corresponding Author: Vasudha Sipayya, Resident, Department of Radiodiagnosis, Datta Meghe Institute of Medical Sciences Wardha, Maharashtra, India, Phone: +917038448184, e-mail: vasudhasipayya@gmail.com
}

\section{INTRODUCTION}

Seizure ${ }^{1}$ is a paroxysmal event due to abnormal, excessive, hyper synchronous discharges from an aggregate of central nervous system (CNS) neurons, and epilepsy is a condition in which a person has recurrent seizures due to some chronic underlying process.

Seizures are an important cause of morbidity and mortality in childhood. It is therefore important to establish accurate diagnosis of seizures and its etiologies to appropriately manage such patients.

About 10.5 million children worldwide are estimated to be suffering from seizures. ${ }^{2}$ In recent years, clinical and etiological diagnosis with better neuroimaging has helped to clarify the vast etiologies of seizures and aid in its management.

A recent meta-analysis ${ }^{3}$ suggests that the prevalence rate in India is 5.59 per 1,000 population with no gender or geographical differences. Reported figures on prevalence rates of seizures in children range from 1.5 to $12.1 / 1,000$, with most figures clustering between 3 and $6 / 1,000$.

As per the International League Against Epilepsy (ILAE 1989), seizures are classified into two categories: Partial seizures and generalized seizures. Because of a significant chance of finding some structural cerebral lesion, an imaging procedure, such as computed tomography (CT) scan is indicated essentially for every child with seizures.

Although magnetic resonance imaging (MRI) is more sensitive and provides better resolution in detecting the cause of seizures, CT is obtained in a few seconds and is less sensitive to patient motion. Long imaging time of MRI requires sedation in most cases and monitoring of the children which is not needed in CT scan. Moreover, $\mathrm{CT}$ is more easily available and affordable. All these factors make CT the preferred tool in imaging of children presenting with seizures.

\section{MATERIALS AND METHODS}

This was a prospective analytical study done from September 2014 to August 2015 in our institute. Eightyfour children who presented to the Department of Radiodiagnosis, Acharya Vinoba Bhave Rural Hospital, Wardha, Maharashtra with history of seizures were 
subjected to CT brain, and history about the onset and frequency of seizures was taken. Axial, coronal, and sagittal sections of each patient were examined.

All children up to the age of 12 years presenting with seizures were taken up for the study.

\section{Inclusion Criteria}

- Children between the age of 0 and 12 years presenting with seizures.

\section{Exclusion Criteria}

- Children in whom CT scan could not be done due to inability to sedate.

The results were presented in number and percentage in tables and figures. Relevant graphs were also used.

\section{RESULTS}

Out of the 84 children presenting with seizures, who underwent CT scan, 55 were males and 29 were females (Table 1).

Maximum number of abnormal scans (Table 2, Graph 1) was found to have congenital anomalies (10), followed by hydrocephalus which was found in nine children. Five children had posttraumatic seizures. Four

Table 1: Sex distribution of all patients

\begin{tabular}{lll}
\hline & Males & Females \\
\hline Number & 55 & 29 \\
Percentage & 65.47 & 34.52 \\
\hline
\end{tabular}

Table 2: Sex distribution of patients with abnormal CT scans

\begin{tabular}{lll}
\hline & Males & Females \\
\hline Number & 24 & 16 \\
Percentage & 60 & 40 \\
\hline
\end{tabular}

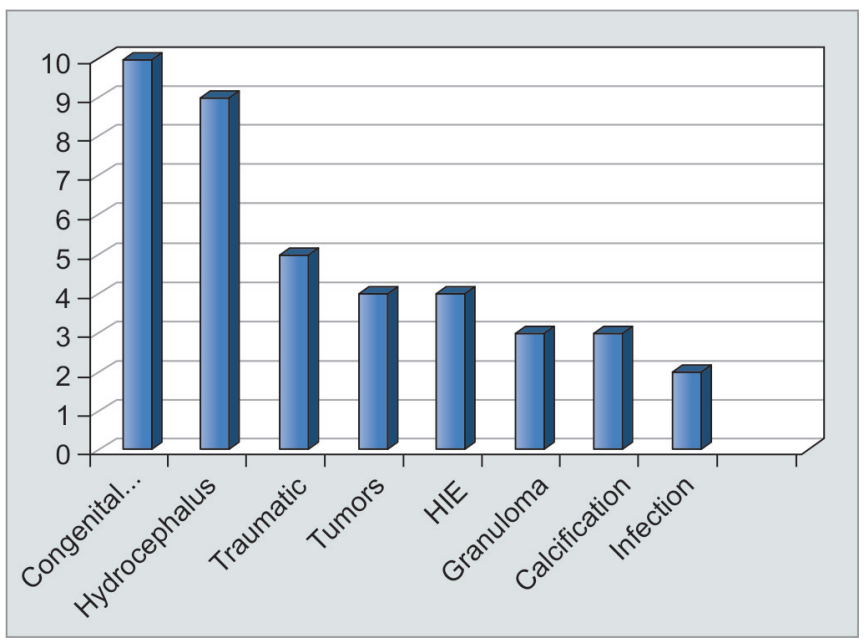

Graph 1: The abnormal CT scan findings
Table 3: Percentage of abnormal CT scan findings

\begin{tabular}{ll}
\hline CT finding & Percentage \\
\hline Congenital anomalies & 11.9 \\
Hydrocephalus & 10.7 \\
Traumatic & 5.9 \\
Tumors & 4.76 \\
Calcification & 3.57 \\
Granuloma & 3.57 \\
Atrophy & 3.57 \\
Infections & 2.38 \\
HIE & 1.19 \\
\hline
\end{tabular}

presented with tumors. Four children showed features of hypoxic ischemic encephalopathy (HIE). Three each had granuloma and calcification. Two children with seizures had infectious causes, which was clinically diagnosed to be meningitis (Table 3).

\section{DISCUSSION}

Seizures are one of the most common presentations of a range of pathologies in pediatric patients. Out of the 84 cases taken up for study, 40 patients showed abnormal CT scans, i.e., $47.6 \%$ of the total scans. There were 55 males $(65.47 \%)$ and 29 females (34.52\%). Out of the 40 abnormal scans, $26(60 \%)$ were males and $16(40 \%)$ were females. Obajimi et al $^{4}$ conducted a study in 103 children presenting with seizures in which $51.5 \%$ of the children had abnormal CT scan; $68.9 \%$ of total patients were males and $31.1 \%$ females.

The most common finding in our study was congenital anomalies comprising 10 cases (11.9\%) of total cases (Table 4). Similar findings were observed by Hsieh et $\mathrm{al}^{5}$ who reported abnormal neuroimaging in children presenting with seizures with $16 \%$ of the patients presenting with congenital anomalies. In another study by Erikkson et $\mathrm{al}^{2}$ the incidence of congenital anomalies was found to be $15 \%$.

We found two cases with Dandy-Walker syndrome (Fig. 1). Both the cases on CT scan showed hypoplastic vermis with communication noted between the 4 th ventricle and the cisterna magna. Clinically, the children had history of seizures with mental retardation.

Table 4: Types of congenital anomalies

\begin{tabular}{ll}
\hline Types & Number of cases \\
\hline Dandy-Walker syndrome & 2 \\
Schizencephaly & 2 \\
Tuberous sclerosis & 2 \\
Arachnoid cyst & 2 \\
Lissencephaly & 1 \\
Corpus callosum agenesis & 1 \\
\hline
\end{tabular}




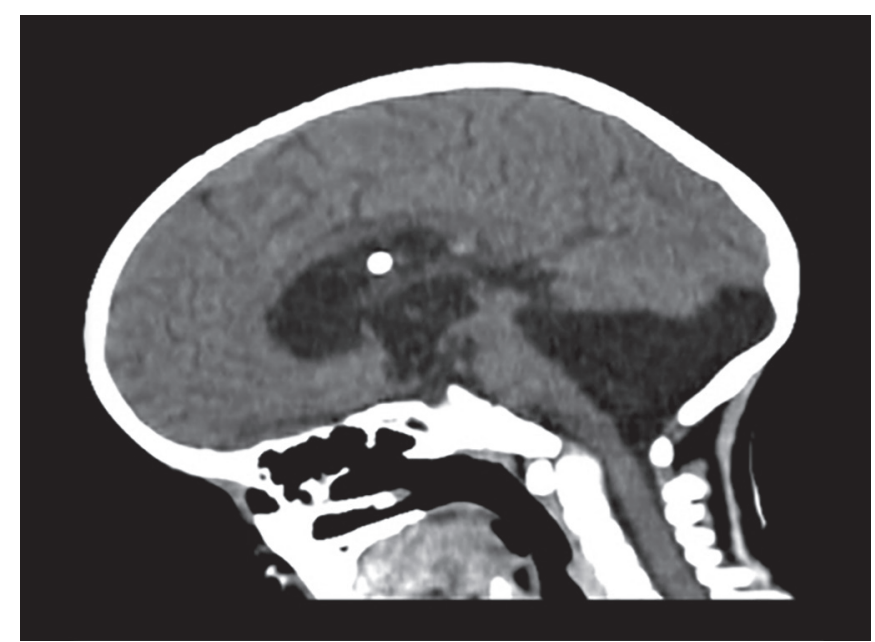

Fig. 1: Nonenhanced CT scan in an 8-year-old male patient with history of seizures since birth and delayed milestones shows an enlarged 4th ventricle communicating with cisterna magna and hypoplastic vermis - Dandy-Walker syndrome

Two children on CT scan showed schizencephaly. Both of them were the open lip type. One patient had bilateral open lip schizencephaly and another had unilateral open lip type.

One child presented with lissencephaly (Fig. 2). The CT scan showed smooth brain with lack of sulci gyri and showed the classical "figure of eight appearance." The child had a history of seizures since birth with mental retardation.

Two children presented with history of seizures since birth and mental retardation, and CT scan showed the presence of calcified subependymal nodules and ill-defined hyper to hypodense areas in the cortex suggestive of tuberous sclerosis. Two children who underwent CT scan for seizures showed the presence of arachnoid cyst. One child on CT scan presenting with neonatal seizures and severe mental retardation showed corpus callosum agenesis.

In our study, nine cases $(9.7 \%)$ presented with hydrocephalus (Table 5). This is similar to the findings in previous studies. Obajimi et $\mathrm{al}^{4}$ reported hydrocephalus as the most common finding in children presenting with seizures and seen in $13.6 \%$ of the children. In our study, out of nine cases of hydrocephalus, six were obstructive and three were nonobstructive.

Five patients (5.9\%) presented with posttraumatic seizures. Three patients had fractures and two had intracranial bleed. Obajimi et al ${ }^{4}$ found $6.8 \%$ of the patients with posttraumatic seizures, which is similar to our finding (Fig. 3). Depressed fractures can compress the underlying

Table 5: Types of hydrocephalus

\begin{tabular}{ll}
\hline Type & Number \\
\hline Communicating & 6 \\
Noncommunicating & 3 \\
\hline
\end{tabular}

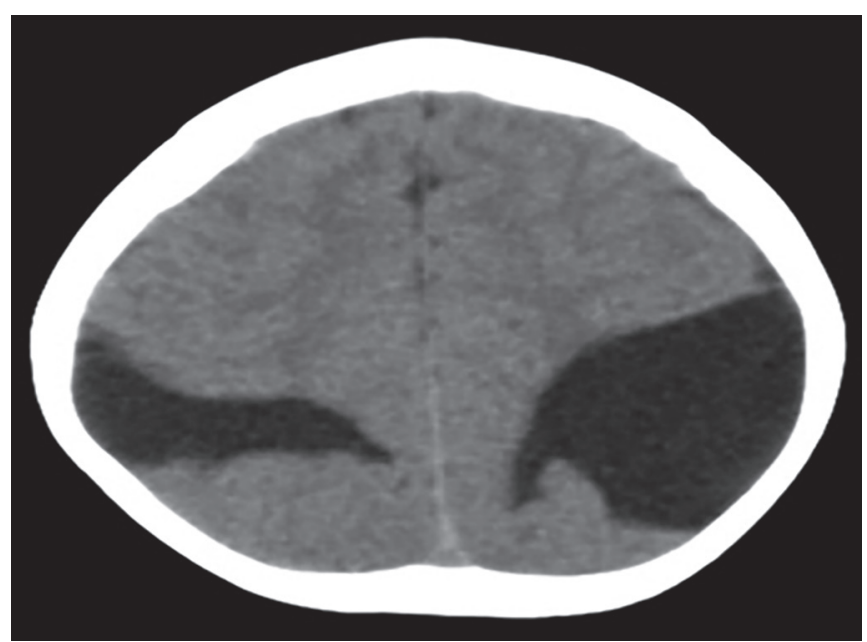

Fig. 2: Non enhanced CT scan of 2-year-old male patient with history of seizures since birth shows reduction in the normal sulcation with small sylvian fissures showing the "figure of eight appearance" with smooth brain - lissencephaly

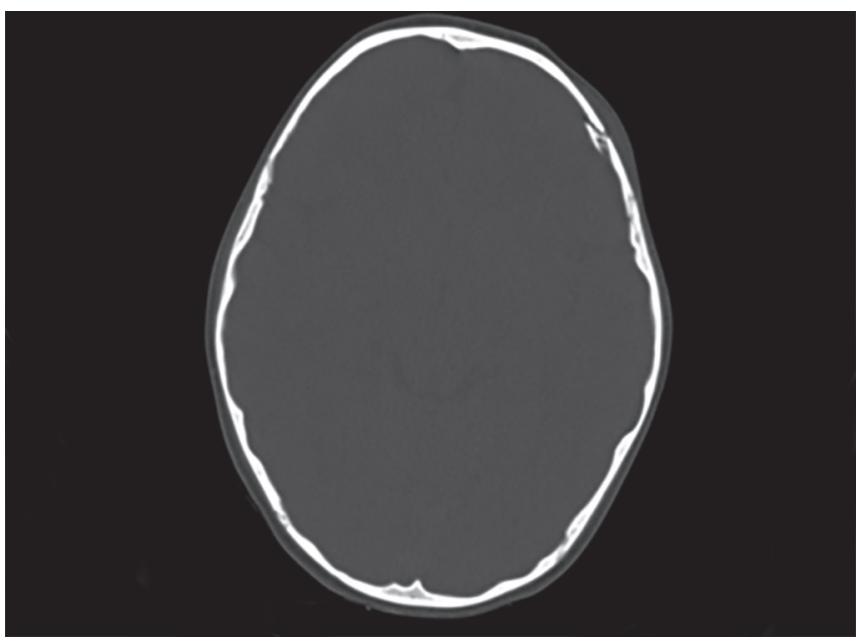

Fig. 3: Nonenhanced CT scan in bone window of a 7-year-old male patient presenting with seizures after history of fall shows depressed displaced fracture of the left parietal bone with the bone fragment protruding into the brain parenchyma - depressed fracture of left parietal bone

brain parenchyma and may cause damage to the neurons, thereby leading to seizures. There is also a high chance of wound infections in such patients.

In our study, we found four patients (4.76\%) presenting with tumors. All four tumors were located in the supratentorial region. This is consistent with the study done by Ibrahim and Appleto ${ }^{6}$ who reported that all tumors causing seizures were supratentorial in location. In our study, one patient presented with subependymal giant cell astrocytoma. This patient was a known case of tuberous sclerosis and was suffering from infantile seizures and mental retardation. We also found two patients with pineoblastoma who presented with seizures of recent onset (Fig. 4). One patient was diagnosed with craniopharyngioma. Erikkson and Koivikko ${ }^{2}$ reported an incidence of $2 \%$. 


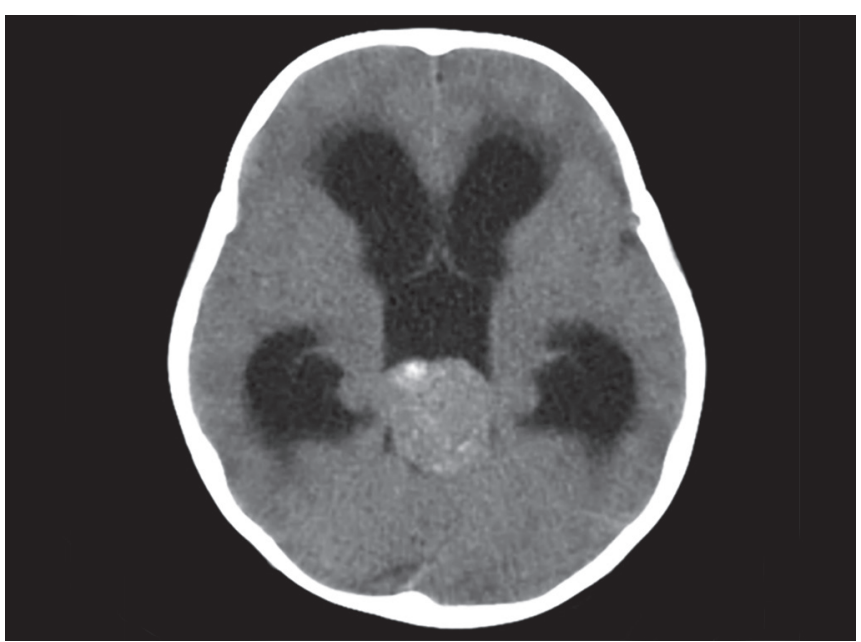

Fig. 4: Nonenhanced CT scan of a 4-year-old female child with history of seizures since 5 months showing a slightly hyperdense midline mass in the pineal region with peripheral calcification. Final histopathological diagnosis - pineoblastoma

Four $(4.76 \%)$ patients were found to have HIE. Eghbalian et $\mathrm{al}^{7}$ found HIE to be the most common cause of neonatal seizures in a study conducted in Iran. The imaging findings differ in preterm and term babies due to immature brain in preterm babies. It can present with germinal matrix - intraventricular hemorrhage or with periventricular leukomalacia. In postnatal and young children, one of the earliest changes on CT scan is hypoattenuation of cerebral hemispheres with relative increased attenuation of the cerebellum. This is known as the reversal sign. ${ }^{8}$

Three patients $(3.5 \%)$ showed areas of calcification in CT scan. Previous studies showed similar incidence of $7.6 \% .{ }^{9}$ Out of the three patients, two were clinically diagnosed to have tuberous sclerosis and the mother of the 3rd patient had history of fever during pregnancy. A diagnosis of TORCH infection was given.

Three (3.5\%) patients also presented with granuloma. Two were in the nodular vesicular stage and one was calcified. Previous studies have shown a wide range of percentage of granuloma. Baheti et $\mathrm{al}^{9}$ reported an incidence of $3.8 \%$ each of neurocysticercosis and tuberculoma. In our study, we did not get any case of tuberculoma. This could be due to small sample size and limiting the upper age limit to 12 years.

Infections were found in two $(2.38 \%)$ patients. Both of them presented with fever, seizures of recent onset and altered sensorium. They were diagnosed to be suffering from meningitis. Erikkson and Koivikko ${ }^{2}$ reported an incidence of $4 \%$ for infections.

\section{LIMITATION}

Although CT scan is more easily available and has shorter scan time, MRI is superior in the diagnosis of most of the pathologies. Malformations of cortical development are best diagnosed on MRI as the gray and white matter junction is visualized clearly. Subtle sulcation-gyration abnormalities are also seen well on MRI. It is also the modality of choice for diagnosis of tumoral extent and for diagnosing presence of any bleed within the mass.

\section{CONCLUSION}

Based on our study, we can conclude that CT scan in children suffering from seizures is extremely useful as almost half of the CT scans were found to be abnormal. It is therefore an excellent tool for screening and also for definitive evaluation of seizures.

It is also useful to monitor the prognosis following treatment since it is cheaper as compared to MRI and is more easily available. It is a short investigation and does not require sedation in most of the cases.

We therefore suggest CT scan to be used as the screening modality in all children presenting with seizures to diagnose the cause of seizures and to monitor the prognosis.

\section{REFERENCES}

1. Berg AT, Berkovic SF, Brodie MJ, Buchhalter J, Cross JH, van Emde Boas W, Engel J, French J, Glauser TA, Mathern GW, et al. Revised terminology and concepts for organization of seizures and epilepsies: report of the ILAE Commission on Classification and Terminology, 2005-2009. Epilepsia 2003 Apr:51(4):676-685.

2. Erikkson KJ, Koivikko MJ. Prevalence, classification, and severity of epilepsy and epileptic syndromes in children. Epilepsia 1997 Dec:38(12):1275-1282.

3. Sridharan R, Murthy BN. Prevalance and pattern of epilepsy in India. Epilepsia 1999 May;40(5):631-636.

4. Obajimi MO, Fatunde OJ, Ogunseyinde AO, Omigbodun OO, Atalabi OM, Joel RU. Computed tomography and childhood seizure disorder in Ibadan. West Afr J Med 2004 Apr-Jun;23(2):167-172.

5. Hsieh DT, Chang T, Tsuchida TN, Vezina LG, Vanderver A, Siedel J, Brown K, Berl MM, Stephens S, Zeitchick A, et al. New onset afebrile seizures in infants. Neurology 2010 Jan 12; 74(2):150-156.

6. Ibrahim K, Appleto R. Seizures as the presenting symptom of brain tumors in children. Seizure 2004 Mar;13(2):108-112.

7. Eghbalian F, Rasuli B, Monsef F. Frequency, causes, and findings of brain CT scans of neonatal seizure at Besat Hospital, Hamadan, Iran. Iran J Child Neurol 2015 Winter;9(1): 56-63.

8. Huang BY, Castillo M. Hypoxic-ischemic brain injury: imaging findings from birth to adulthood. Radiographics 2008 Mar-Apr;28(2):417-439.

9. Baheti R, Gupta BD, Baheti R. A study of CT and EEG findings in patients with generalised or partial seizures in Western Rajasthan. J Indian Acad Clin Med 2003;4(1):25-29. 